\title{
Novel Treatment Options for Neuroendocrine Tumors
}

\author{
Presented by Matthew H. Kulke, MD, MMSc
}

\begin{abstract}
Neuroendocrine tumors (NETs) are not as rare as once thought, with a current prevalence that is estimated to be higher than that of many other gastrointestinal tumors. Multiple treatment options are available for these tumors, which are categorized according to their histology and site of origin. For patients with metastatic disease, somatostatin analogues are often the initial treatment, with other options considered when these drugs fail.
\end{abstract}

J Natl Compr Canc Netw 2017;15(5.5):737-739

Understanding of neuroendocrine tumors (NETs) has rapidly evolved over the past several years. With this new knowledge, diagnoses have increased over the past few decades. NETs are thought to arise from cells in the diffuse neuroendocrine system throughout the body. Typically, they are well-differentiated and grow more slowly than many other tumor types. In many cases, NETs are resistant to standard cytotoxic chemotherapy. Although most are nonfunctional, NETs have the ability to secrete neuropeptides that may result in symptoms of hormone hypersecretion (functional).

A current estimate of NET prevalence is 103,312 individuals in North America, which is higher than that of many other gastrointestinal tumors, including esophageal, pancreatic, gastric, and hepatobiliary cancers.

Most NETs are well-differentiated, but the histology of these tumors has a broad spectrum, explained Matthew H. Kulke, MD, MMSc, Director, Neuroendocrine and Carcinoid Tumor Program, Dana-Farber Cancer Institute, at the NCCN 22nd Annual Conference. Classification of NETs is based on differentiation and grade, he continued. Well-differentiated tumors are either low-grade (grade 1) or intermediate-grade (grade 2 ); although poorly differentiated tumors are generally considered grade 3. Grade 2 tumors tend to be more

Presented by Matthew H. Kulke, MD, MMSc, Dana-Farber Cancer Institute, Boston, Massachusetts.

Dr. Kulke has disclosed that he receives consulting fees/honoraria from Ipsen, Lexicon Pharmaceuticals, Inc., and Novartis Pharmaceuticals Corporation.

Correspondence: Matthew H. Kulke, MD, MMSc, Dana-Farber Cancer Institute, 450 Brookline Avenue, Boston, MA 02215-5450.

E-mail: matthew_kulke@dfci.harvard.edu aggressive than grade 1, and the prognosis is better for grade 1 tumors than for grades 2 and 3. "The classification scheme continues to be debated," and may be updated in the next few years, he said.

For example, a study of 252 patients with poorly differentiated NETs (grade 3 ) treated with platinum/etoposide found that tumors with a $\mathrm{Ki}-67$ index $>55 \% \mathrm{had}$ a much higher response rate. ${ }^{1}$ Thus, the $>55 \%$ cutoff for Ki-67 may be more appropriate for poorly differentiated tumors when considering treatment selection, Dr. Kulke noted. Tumors with a Ki-67 index $<55 \%$ may respond more favorably to treatment described for welldifferentiated tumors.

\section{Pancreatic NETs}

NETs are categorized according to site of origin: pancreatic (islet cell tumors) or carcinoid. Depending on the data set, pancreatic NETs (panNETs) constitute between $5 \%$ and $28 \%$ of all NETs. panNETs can be associated with symptoms of hormone hypersecretion, though the majority are often clinically silent and not diagnosed until they are widely metastatic (Figure 1).

Currently, the only curative treatment is surgical resection, with the type of procedure dependent on tumor size and location. The prognosis is good when complete resection is performed.

Patients with functional tumors should initially be stabilized by managing hormonal syndromes. For example, patients with glucagonoma often respond favorably to somatostatin analogues (SSAs), as do patients with tumors secreting vasoactive intestinal peptides (VIPomas). 


\section{Kulke}

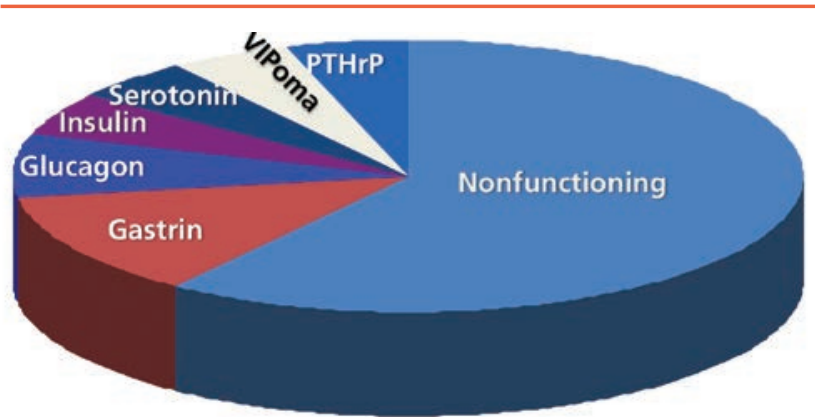

Figure 1. Pancreatic neuroendocrine tumors distribution of tumor subtypes.

Abbreviations: PTHrP, parathyroid hormone-related protein; VIPoma, vasoactive intestinal peptide.

From Strosberg J, Gardner N, Kvols L. Survival and prognostic factor analysis in patients with metastatic pancreatic endocrine carcinomas. Pancreas 2009;38:255-258. Reprinted with permission from Wolters Kluwer Health, Inc. @2009.

Patients with gastrinoma should be treated with proton pump inhibitors and, in later stages, SSAs.

\section{Carcinoid Tumors}

Carcinoid NETs can arise in the foregut (33\%), midgut $(34 \%)$, and hindgut (14\%); examples include bronchial, appendiceal, rectal, and small bowel carcinoid tumors.

Patients with bronchial carcinoid tumors present with hemoptysis, cough, and wheezing. The prognosis of these tumors depends on histology; patients with resected typical bronchial carcinoid tumors generally have a good prognosis, whereas those that are atypical have a higher rate of recurrence. Appendiceal carcinoids are relatively common, usually occur in younger patients, and present with acute appendicitis. Smaller tumors $(<2 \mathrm{~cm})$ have a good prognosis when treated with simple appendectomy; tumors $>2 \mathrm{~cm}$ should generally be managed with a right colectomy. Prognosis and management for patients with rectal carcinoids is also determined by tumor size: $<2 \mathrm{~cm}$ versus $>2 \mathrm{~cm}$.

Small bowel carcinoids are another common subtype, and are difficult to diagnose at an early stage. These tumors arise in the muscle wall of the intestine and are not readily visible on endoscopy. They are associated with mesenteric fibrosis, and patients can experience abdominal discomfort for years before they are finally diagnosed, often with metastatic disease.

\section{Metastatic NETs}

Studies evaluating survival durations for patients with metastatic NETs vary widely in their estimates. According to SEER estimates, the median survival for metastatic panNET is 2 years and for metastatic small bowel carcinoid is 4.6 years, whereas a singleinstitution database from Dana-Farber Cancer Center reported survival rates of 5.9 and 10.1 years, respectively, Dr. Kulke shared. Although survival estimates for individual patients may differ, "In most cases, metastatic disease is fatal," he said.

Such patients may nevertheless benefit from a range of treatment options for metastatic NETs, which include SSAs, liver-directed therapy, cytotoxic chemotherapy, and targeted therapy.

NETs often express somatostatin receptors and can be visualized with somatostatin receptor-based imaging. A new form of somatostatin receptor-based imaging, the Gallium (68Ga-DOTATATE) PET scan, has been added to the 2017 NCCN Clinical Practice Guidelines in Oncology (NCCN Guidelines) for NETs. Such imaging can help define the extent of metastatic disease, and by confirming the presence of somatostatin receptors, can help determine whether SSAs should be used for treatment.

Patients with metastatic NETs may develop carcinoid syndrome, which is caused by secretion of serotonin and other neuropeptides into the systemic circulation, and is manifested by episodic flushing and diarrhea. SSAs are generally used for the initial treatment of carcinoid syndrome, but their efficacy can decrease over time.

Telotristat ethyl, a tryptophan hydroxylase (TPH) inhibitor, is a new treatment option for patients with carcinoid syndrome. Telotristat ethyl does not cross the blood-brain barrier and is associated with minimal central nervous system activity. The international randomized TELESTAR phase III study showed that telotristat significantly reduced the frequency of bowel movements compared with placebo $(P<.001)$, and was also associated with reductions in urinary 5 -hydroxyindole acetic acid, a serotonin metabolite. ${ }^{2}$

Telotristat ethyl was approved by the FDA on February 28, 2017, and "will be considered for inclusion in the NCCN Guidelines as a treatment option for carcinoid syndrome," Dr. Kulke explained.

In the 2017 NCCN Guidelines, the SSAs octreotide and lanreotide are also recommended for the control of tumor growth in patients with metastatic 
NETs. They can be considered for asymptomatic patients with a low tumor burden, and are recommended for use in patients with disease-related symptoms or a clinically significant tumor burden (ie, unresectable disease and/or distant metastases). These drugs are generally well tolerated.

\section{Progression on SSAs}

\section{Patients With Hepatic Predominant Disease}

Patients with metastatic NETs and hepatic-predominant disease may benefit from hepatic-directed therapy. Recommended liver-directed therapies for patients with liver-predominant disease include hepatic resection and hepatic artery embolization (unresectable disease). Liver transplantation is only considered for highly selected patients, Dr. Kulke noted. Although institutional series of patients undergoing liver transplant generally report that 5-year survival is $>50 \%$, most patients who undergo a liver transplant will nevertheless develop recurrence.

\section{panNETs}

Cytotoxic chemotherapy is an option for patients with advanced panNETs who experience disease progression on SSAs. Streptozocin-based therapy has been shown to achieve response rates of $30 \%$ to $40 \%$ in panNETs, but this intravenously administered alkylating agent can be associated with toxicity and requires 5 days of in-office treatment. Temozolomide, an oral alkylating agent, has gained favor as a chemotherapeutic agent for panNETs. Reported response rates to temozolomide-based therapy in small retrospective series and prospective phase II studies range from $8 \%$ as a single-agent to $70 \%$ in combination with capecitabine.
"We still don't know the best way to use temozolomide. As a single agent? In combination? With what drugs?" Dr. Kulke continued. ECOG E2211 is an ongoing randomized study in patients with advanced panNETs evaluating temozolomide alone versus combination temozolomide and capecitabine. ${ }^{3}$

Molecularly targeted agents also play an important role in the management of advanced panNETs. In a placebo-controlled trial, sunitinib improved median progression-free survival (PFS) to 11.4 months versus 5.5 months with placebo $(P=.0001) .{ }^{4}$ In the RADIANT trial, everolimus also significantly improved PFS versus placebo in advanced panNETs. ${ }^{5}$ These studies led to FDA approval of everolimus and sunitinib for NETs, and both drugs remain important treatment options for patients with advanced disease.

\section{Carcinoid}

The 2017 NCCN Guidelines recommend everolimus for the treatment of advanced carcinoid NETs after progression on SSAs, based on the results of the RADIANT-4 study, which demonstrated superior PFS in patients treated with everolimus compared with placebo in patients with advanced carcinoid tumors. Everolimus is FDA-approved for both advanced carcinoid NETs and panNETs. Cytotoxic chemotherapy should be considered if no other options are feasible.

Looking toward the future, studies are ongoing to identify molecular and genetic predictors of response, novel targets, and novel agents. Agents under study include peptide receptor radionuclide therapy, cabozantinib, and immune checkpoint inhibitors. Investigators are examining the genomic landscape and immune environment of NETs, and how they can inform treatment selection.

\section{References}

1. Sorbye H, Welin S, Langer SW, et al. Predictive and prognostic factors for treatment and survival in 305 patients with advanced gastrointestinal neuroendocrine carcinoma (WHO G3): the NORDIC NEC study. Ann Oncol 2013;24:152-160.

2. Kulke MH, Horsch D, Caplin ME, et al. Telotristat ethyl, a tryptophan hydroxylase inhibitor for the treatment of carcinoid syndrome. J Clin Oncol 2017;35:14-23.

3. Kunz PL, Catalano PJ, Nimeiri HS, et al. A randomized study of temozolomide and capecitabine in patients with advanced pancreatic neuroendocrine tumors: a trial of the ECOG-ACRIN Cancer Research Group (E2211) [abstract]. J Clin Oncol 2015;33(Suppl):Abstract TPS4145.

4. Raymond E, Dahan L, Raoul JL, et al. Sunitinib malate for the treatment of pancreatic neuroendocrine tumors. N Engl J Med 2011;364:501-513.

5. Yao JC, Shah MK, Ito $\mathrm{T}$, et al. Everolimus for advanced pancreatic neuroendocrine tumors. N Engl J Med 2011;364:514-523. 